\title{
To Beep or not to Beep: On the Influence of Some Interaction Design Variables on Its Performance
}

\author{
Ulrich J. Bröckl ${ }^{1}$, Belém Priego Sánchez ${ }^{2}$ \\ ${ }^{1}$ Hochschule Karlsruhe, \\ Fakultät für Informatik und Wirtschaftsinformatik, Germany \\ ${ }^{2}$ Universidad Autónoma Metropolitana unidad Azcapotzalco, \\ Departamento de Sistemas, unidad Azcapotzalco, Mexico
}

Ulrich.Broeckl@hs-karlsruhe.de, abps@azc.uam.mx

\begin{abstract}
During the construction and development of graphical interfaces, it is important to decide among a huge number of possible feedbacks (colors, sounds, etc.) with graphical variants (circular or rectangular shapes) and graphical behaviors (Close- and X-Button in title-bar e.g. to close a window). In this paper, the results of a long-term experiment comparing mouse- and touch-based interaction are presented. The aim of this work is to compare design variables of interaction in order to explore the influence of these variables on the interactions performance. The obtained results, for mouse interaction, reveal that only one studied design variable (clicking or not when reaching a target) is independent from target size. Color feedback showed to have negative influence for large objects and positive influence for small objects. For small targets circle-shaped targets and sound feedback proved to be negative, on the contrary to touch interaction. Here sound feedback saves for small objects $14.5 \%$ of interaction time. Finally the best results obtained are condensed to simple to apply design rules.
\end{abstract}

Keywords. Graphical interfaces, variables of interaction, mouse interaction.

\section{Introduction}

When building a Graphical User Interface, designers need to decide for a huge variety of possible feedbacks and decide among many graphical variants. Besides of graphical feedbacks like using color as a feedback for successful interaction sound feedback may be used there. Other graphical decisions are whether to take rectangular, or more fashionable, circular targets. Or whether to offer several targets for the same functionality (Close- and X-Button in title-bar e.g. to close a window) or restrict the design to a single target for each functionality.

These design variables are of interest in several areas. Firstly, GUI- and Web-Designers need that knowledge when designing new controls, for instance 
new button or menu control elements. Secondly and more generally the correct decision of these design variables also may support better design of any $2 \mathrm{D}$ pointing instrument based interaction since large control elements like panels and boxes were inestigated too. Thirdly the results presented in that article may serve GUI- and Web-Designers to calculate the operating times in advance and thus to predict or compare the performance of designs with freely chosen variables.

In this work the results of a long-term experiment comparing mouse- and touch-based interaction are presented. Especially, the influence of above mentioned design variables is studied. Fitts' Law was used to differentiate among small and large targets. The results reveal for mouse interaction that only one studied design variable (clicking or not when reaching a target) is independent from target size. Color feedback showed to have negative influence for large objects and positive influence for small objects. For small targets circle-shaped targets and sound feedback proved to be negative. On the contrary to touch interaction. Here sound feedback saves for small objects: $14.5 \%$ of interaction time. Finally the won results are condensed to simple to apply design rules.

\section{Related Work}

A computer is made up of different devices that allow a user to interact and control it. In this paper, it is carried out a study of the "mouse", a device that allows a computer user to control a screen pointer or cursor and to do click in screen positions that determine a given flow of information. During the $60 \mathrm{~s}$, one of the pioneers of the human-computer interaction area, Douglas C. Engelbart invented the mouse device with the help of Bill K. English.

The first mention of the term "mouse" in the literature, as an input device, was made when the input device at Stanford Research Institute, Menlo Park, CA developed by [4] was created. A comparison of this input device with other ones has been done throughout the years. For example, in 1967 a comparison among mouse, joystick and a fight pen was performed by [4]. The aim of that comparison was to select characters and words in the computer screen; the results obtained shown that the use of the mouse device is faster than the other ones. Other comparisons against the mouse device has been done, for example, with rare-controlled isometric joystick, step keys and text keys [1], or with other input devices such as touch screens, panel and keywords [8], with graphic tablet and trackball [10], with absolute touchpad, relative touchpad, trackball, displacement joystick, and force joystick [5], with high precision touch screens [18], with a pen device [2], with the finger-controlled isometric joystick [12], with touchpad and multitouch input technologies [19], just to mention some of the diverse literature dedicated to performance comparison of the mouse device with respect to other ones. Here we can see the numerous research works in which the mouse device is compared with different input devices, however, it is also important to mention the manner in which the mouse device has influenced the use and construction of graphical interfaces. Perry and Voelcker [15], for instance, present a perspective 
of the development of the mouse device and user-friendly interfaces. A button size and spacing on touch screen buttons experiment was made by [9]. They compared performance and input accuracy between older adults and younger adults. Their results have shown that younger adults required significantly less amount of time to complete a given input task. Input accuracy did not show significant different between older and younger adults. Authors found not any button size or spacing configuration where younger or older adults were stronger or weaker with. However, although spacing difference did not affect time performance, it significantly affects input accuracy.

\section{Methods}

\subsection{Experimental Design}

The experiments were conducted in fairs, exhibitions or laboratory sessions using a Windows program as shown in the Figure 1. After a short poster-based introduction into the experiments and privacy issues, the participants performed the test undisturbed.

When the program is started the user is first asked to convey some sociodemographic and basic data (age, gender, device type) in a pop-up window. Furthermore, binary test variables were set in that window: sound feedback on/off, color feedback on/off, circular or rectangular targets, and display of one or several targets at once (only one of these needed to be hit). These are considered as test variables for this article.

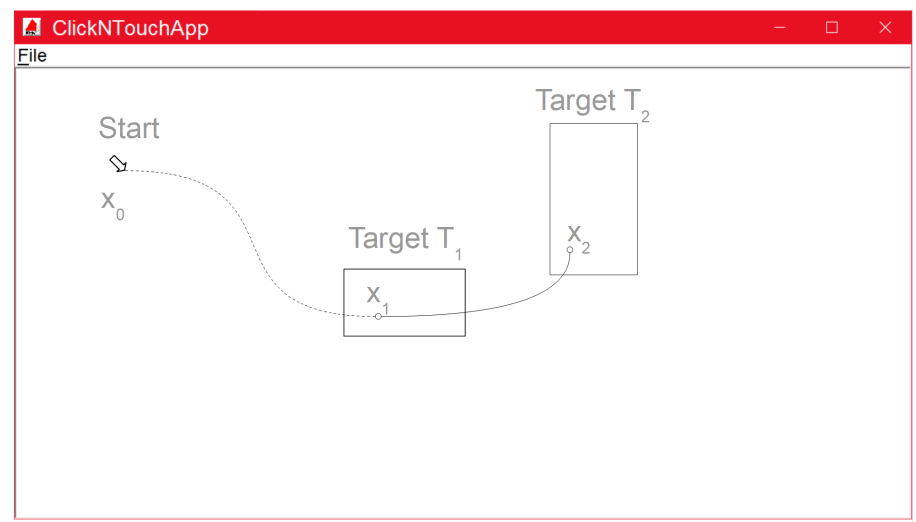

Fig. 1. Basic experimental design.

Right after that basic data acquisition, the user is shown targets by the program (rectangles or circles, see Figure 1 and the user clicks or touches on these. As soon as the target is hit, and only if it is hit, the next target is shown. So in Figure 1 the user starts at position $x_{0}$, begins to move to position $x_{1}$ and 
clicks in target $T 1$. Directly after that Target $T 1$ vanishes and Target $T 2$ is shown. Thereafter the user moves from $x_{1}$ to $x_{2}$ and clicks in Target $T 2$.

The picture's inscriptions for explanation in the picture $\left(\right.$ Start,$\left.x_{0},\right)$ and the mouse-traces were not shown in the real experiment. The application was opened in full-screen mode, so the title bar and File-menu were not visible. Actually only the targets and the mouse pointer became visible. Depending on the experiments context (sessions in fairs and exhibitions or laboratory sessions) the users had to hit between 50 up to 300 different targets (average 99.6 targets). In total 15,689 hits were stored, 35 were peaked out before $(0.22 \%)$ since the users used more than $3000 \mathrm{~ms}$ to hit the target.

The targets areas were sized randomly between 440 and 84213 pixels $(0.012 \%$ $4.4 \%$ of the screen's area). The $\mathrm{x}$ - and $\mathrm{y}$-positions for the next targets were as well chosen randomly; the cases that the next target does not move or does move only little in comparison to the last target was not excluded.

\subsection{Subjects}

The experiments took place from 2013 to 2017; the subjects used either standard PCs with HDTV Monitors (27\%) or smaller Laptops (73\%, Asus Slate and Microsoft Surface) where the touch capacities of these were used for touch/mouse comparisons. In total 163 experiments were conducted (38 female, 125 male). Since the experiments took place several times on fairs and exhibitions a wider range of ages is covered (average age 31 years, standard deviation 18 years).

\subsection{Analysis}

The data of the experiments was stored as CSV-files on disk and afterwards an import tool was used to integrate all data into an SQL database. SQL queries were used to peak out and to calculate the Fitts Law regression parameters as defined in [10].

In order to check the significance of the differences furthermore the average interaction time and its standard deviation for the lower and upper half of the ID-interval was calculated. In the variable-comparing plots (e.g. in Figure 3) these IDs are depicted by diamond symbols.

The R program for Statistical Computing was used as an SQL interface and it was used to calculate the significance of differences using Rs Welch Modified Two-Sample t-Test. Furthermore R was used to plot the results.

Fitts Law. The analysis of the given data is based on Fitts Law [6]. There are several variants of that law available, see [3] for details on the ongoing discussion. We used the definition of the Index of Difficulty (ID) as being proposed in [10] since it is standardized in [13].

That definition of ID and the measured interaction times (in ms) were used to calculate Fitts Law linear regression as shown in the figure 2. 


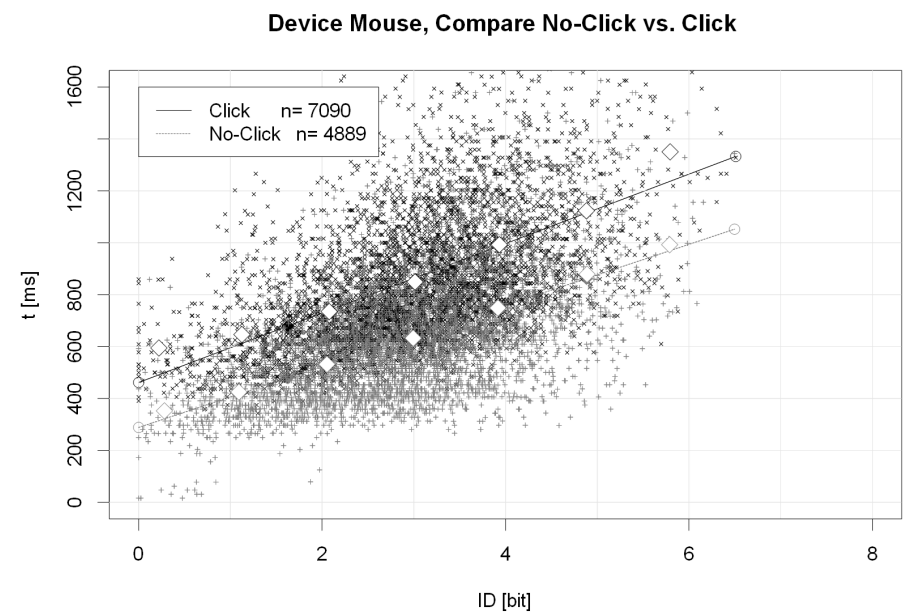

Fig. 2. Scatter plot to compare click and no-click interaction all scatters.

Device Mouse, Compare No-Click vs. Click

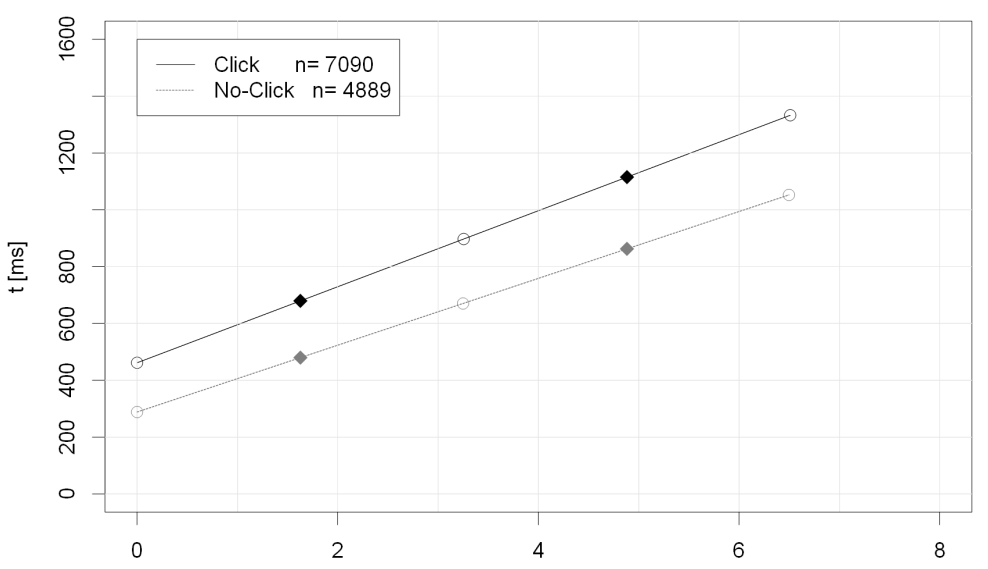

Fig. 3. Regression plot to compare click and no-click interaction.

The small $\mathrm{x}$ - and +-symbols depict a single interaction. The regression lines are ended by circle-symbols. In order to show the linearity of the averaged IDs these are shown in that figure too (white diamond symbols). For the sake of clearness these and the $\mathrm{x}$ - and +-symbols for a single interaction are omitted in the subsequent figures.

Significance Tests. Figure 3 shows that reduced variant of the scatter plot in Figure 2. The diamond symbols depict the points on the $\mathrm{x}$-axis (thus ID-values) for which a significance tests of the differences between the different outcomes 
of the studied variable(s) were conducted. This was done by using R's Welch Modified Two-Sample t-Test for both pairs of diamond symbols.

For the left pair $(I D=1.63 b i t)$ we measured an average width and height of the targets of $113 \times 75$ Pixels $(0.46 \%$ of screen size). For the right pair $(I D=$ $4.88 b i t)$ we have $79 \times 61$ pixels $(0.19 \%)$. The left pair is denoted from now on as "Large Targets" and the right pair as "Small Targets".

\section{Device Mouse, Compare Color Feedback Off or On}

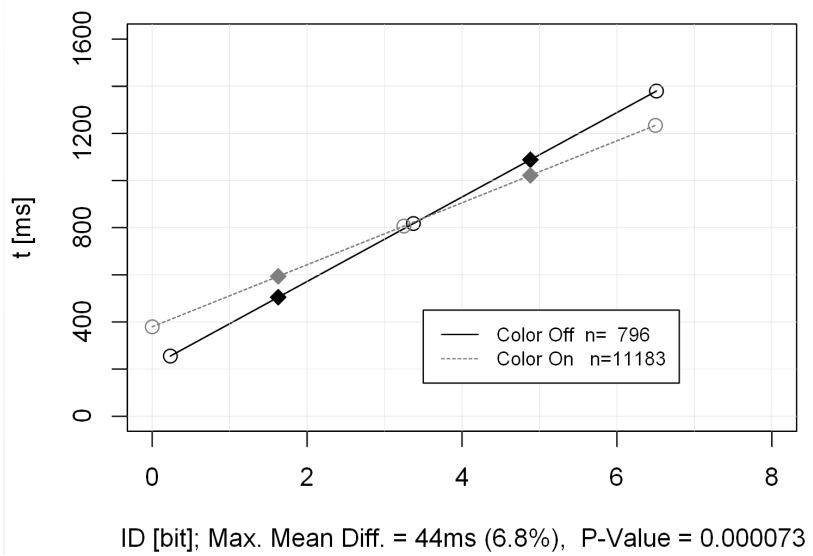

Fig. 4. Using color feedback.

\section{Results}

For all investigated devices (touch and mouse) and variables (circular or rectangular targets, sound feedback on/off, click needed or not, single or multiple targets on screen, color feedback on/off) the Fitts Law regressions and the average interaction times for small and large targets were calculated. Furthermore the p-value of the t-test was computed to check if the differences of the averages are significant. In the following paragraphs only those variables are presented, for which the average interaction times for small and large targets differ more than $6 \%$ and have a $p-$ value $<0.02$.

\subsection{Mouse Interaction}

Variables of Influence for Small and Large Targets. The only variable that exhibited advantages independently of the target size is shown in Figure 3. The difference here is that the users do not have to click in order to get the next target. That difference is for small targets $27.40 \%$, for large targets as much as $33.20 \%$. 
This advantage is already used in several occasions in current GUIs: on the one hand for onmouseover-events in web pages [16] or for tooltips, e.g. in Microsofts ribbons [11]. Here the mouse interaction is used to trigger a functionality that does not cause an irreversible action. On the other hand, the design study in [7] shows that a click-free interaction is possible also for irreversible action - though it exhibits lacks in conformity with user expectations and suitability for learning.

For mouse interaction the usage of color feedback influences the performance depending on the target's size: small targets are clicked $6.80 \%$ faster. If we omit color feedback, large targets gain $5.60 \%$ performance (Fig. 4).

For mouse interaction the usage of several targets simultaneously showed near to no influence on the interactions performance, especially if large targets are considered. Here we noted only a difference of $3.9 \%$ Fig. 5).

\section{Device Mouse, Compare Single- vs. Multi-Target}

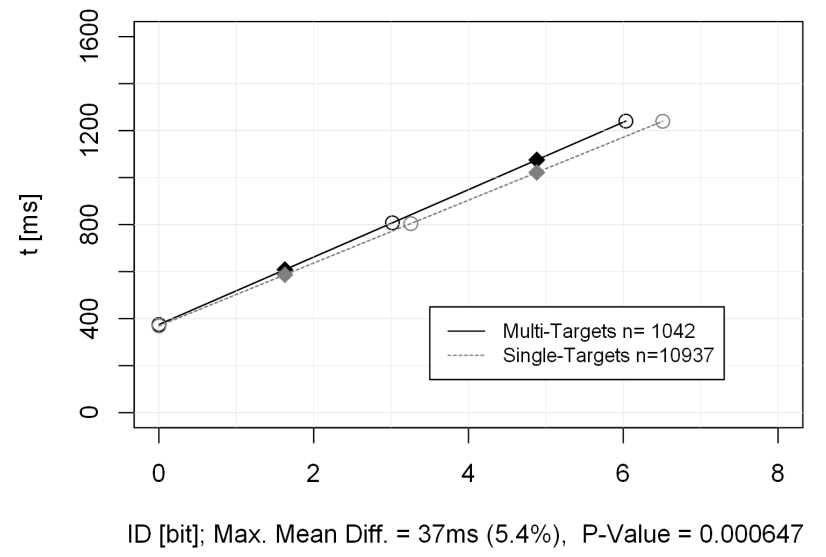

Fig. 5. Using multiple clickable targets.

Variables of Influence for Small Targets. With mouse interaction we detected a difference for small objects of $7.5 \%$ of interaction time when circles are not used instead of rectangles (Fig. 6)

For small targets using sound feedback proved to be negative: $6.8 \%$ of performance are lost. For large targets still $2.7 \%$ are lost (Fig. 7).

Combination of Variables of High Influence. In order to study the influence of the combinations of several variables we tested all possible combinations. The only exception here was the variable whether the user needs to click to get the next target. This variable was excluded since the large influence of that variable heavily shadows the influence of the other variables. 
Device Mouse, Compare Circles vs. Rectangles

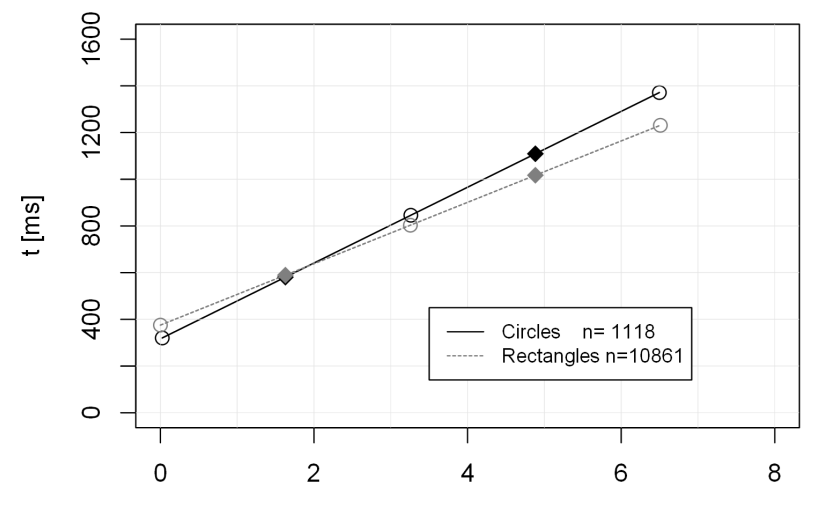

ID [bit]; Max. Mean Diff. $=71 \mathrm{~ms}(7.5 \%), \quad$ P-Value $=0.000007$

Fig. 6. Circles for interaction

Device Mouse, Compare Sound vs. Silence

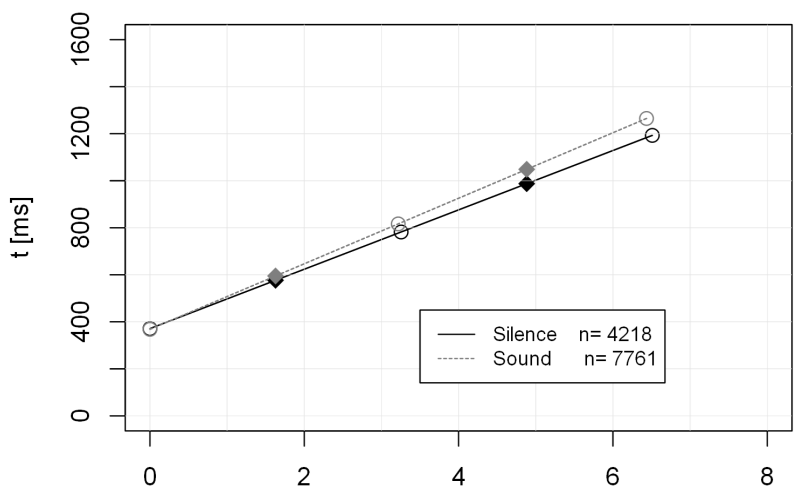

ID [bit]; Max. Mean Diff. $=62 \mathrm{~ms}(6.8 \%), \quad$-Value $=0.000000$

Fig. 7. Sound feedback.

The best combination was using color and multiple targets, and restrict from using sound. That delivered for small targets an advantage of $23.2 \%$ and for large targets $19.8 \%$ (Fig. 8).

\subsection{Touch Interaction}

Variables of High Influence for Small Targets. For touch interaction we detected a difference for small objects: $14.5 \%$ of interaction time can be won if we use sound feedback for small objects. This is a clear difference to mouse 
To Beep or not to Beep: On the Influence of some Interaction Design Variables onto Its Performance

Device Mouse, Compare Sound off, Color, Multi-Target

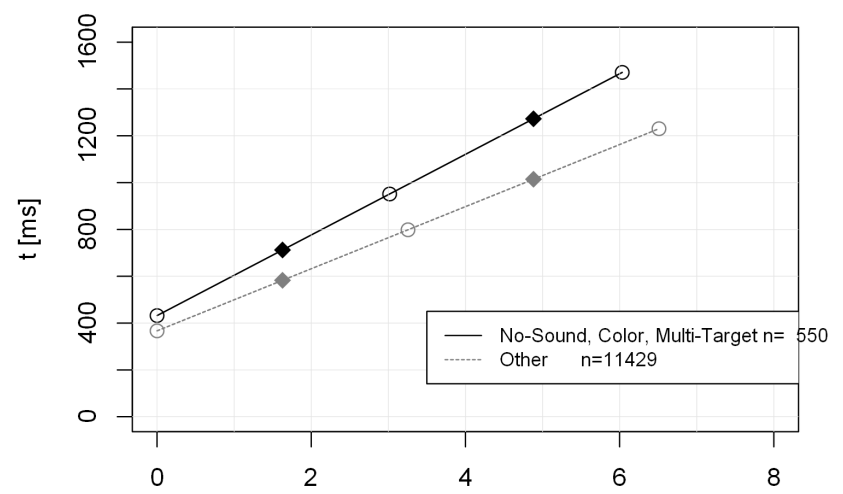

ID [bit]; Max. Mean Diff. $=175 \mathrm{~ms}(23.2 \%), \quad P-$ Value $=0.000000$

Fig. 8. Sound feedback for touch interaction.

Device Touch, Compare Sound vs. Silence

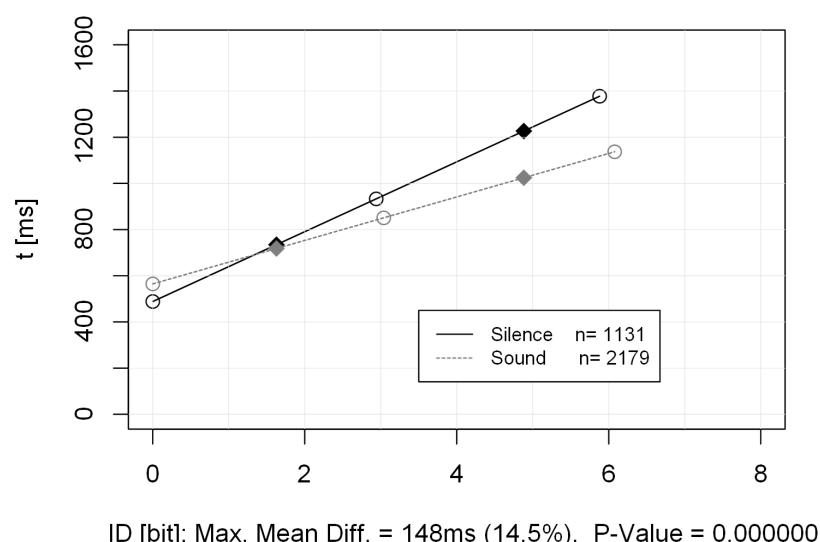

Fig. 9. Sound feedback for touch interaction.

interaction: in paragraph 4.1 .2 we saw a performance loss of $6.8 \%$ if sound is used (Fig. 9).

For other variables (circular or rectangular targets, single or multiple targets on screen, color feedback) we could find no clearly significant influences, probably due to insufficient number of experiments. 


\section{Discussion and Conclusion}

One meta result of this study is the fact that when studying influences of different feedbacks the size of the targets matters. Only one variable (whether to click or not for the next target) was not influenced by the size. Others variables like using rectangles instead of circles as targets only have positive influence if the targets are small. Other variables like color feedback heavily depend on the targets' sizes: color feedback only serves for better performance for small targets, large targets are influenced negatively.

The variables also proved to be interdependent: Unfortunately, when studying the different variable combinations a simple combination of the best outcomes of each single variable in order to obtain an optimal combination did not work.

Finally, we found one variable (sound feedback) that delivered contradictive results for mouse- and touch-based interaction. Here design strategies like Mobile First as being proposed for example in [17] should be reconsidered.

To sum it up: Following main rules can be derived from the study:

- Use sound feedback for touch interaction, avoid it for mouse interaction.

- Better do not use circular targets for small targets.

- For mouse interaction a combination of color feedback, silence and using multiple targets worked best.

- If possible think about avoiding clicks to trigger an intended functionality. One hot candidate seen by the authors to do so: the annoying but necessary cookie messages in websites could be switch off by a simple onmouseoverevent. See for instance [14] for details on cookies law.

For some variables of touch interaction there are needed more experiments in future to identify further significant differences.

\section{References}

1. Card, S.K., English, W.K., Burr, B.J.: Evaluation of Mouse, Rate-Controlled Isometric Joystick, Step Keys, and Text Keys for Text Selection on a CRT. Ergonomics 21(8), 601-613 (1978), http://dx.doi.org/10.1080/00140137808931762

2. Charness, N., Bosman, E.A., Elliott, R.G.: Senior-Friendly Input Devices: Is the Pen Mightier than the Mouse? In: 103rd Annual Convention of the American Psychological Association Meeting. New York, New York (Aug 1995)

3. Drewes, H.: Only one fitts' law formula please! In: CHI '10 Extended Abstracts on Human Factors in Computing Systems. pp. 2813-2822. CHI EA '10, ACM, New York, NY, USA (2010), http://doi.acm.org/10.1145/1753846.1753867

4. Engelbart, D.C., English, W.K.: A research center for augmenting human intellect. In: Proceedings of the December 9-11, 1968, Fall Joint Computer Conference, Part I. pp. 395-410. AFIPS '68 (Fall, part I), ACM, New York, NY, USA (1968), http: //doi.acm.org/10.1145/1476589.1476645

5. Epps, B.W.: Comparison of six cursor control devices based on fitts' law models. Proceedings of the Human Factors Society Annual Meeting 30(4), 327-331 (1986)

6. Fitts, P.M.: The information capacity of the human motor system in controlling the amplitude of movement. Journal of Experimental PSychology 74, 381-391 (1954) 
7. Frank, A.: Dontclick. urlhttp://www.dontclick.it/ (2017), accessed 12-09-2017

8. Karat, J., M.J.E.A.M.: A comparison of menu selection techniques: touch, panel, mouse, and keyboard. In: International Journal of Man-Machine Studies. pp. 73-88

9. KIM, J.M., ISHIBASHI, K., IWANAGA, K.: The effect of invisible touch sensitive area on target acquisition. Proceedings of the Annual Meeting of Japan Ergonomics Society 48spl, 188-189 (2012)

10. MacKenzie, I.S., Buxton, W.: Extending fitts' law to two-dimensional tasks. In: Proceedings of the SIGCHI Conference on Human Factors in Computing Systems. pp. 219-226. CHI '92, ACM, New York, NY, USA (1992), http://doi.acm.org/ $10.1145 / 142750.142794$

11. Microsoft: "ribbons" windows dev center. urlhttps://msdn.microsoft.com/enus/library/windows/desktop/dn742393(v=vs.85).aspx (2017), accessed 12-09-2017

12. Mithal, A.K., Douglas, S.A.: Differences in movement microstructure of the mouse and the finger-controlled isometric joystick. In: Proceedings of the SIGCHI Conference on Human Factors in Computing Systems. pp. 300-307. CHI '96, ACM, New York, NY, USA (1996), http://doi.acm.org/10.1145/238386.238533

13. Natapov, D., Castellucci, S.J., MacKenzie, I.S.: Iso 9241-9 evaluation of video game controllers. In: Proceedings of Graphics Interface 2009. pp. 223-230. GI '09, Canadian Information Processing Society, Toronto, Ont., Canada, Canada (2009), http://dl . acm.org/citation. cfm?id=1555880.1555930

14. Optanon: The cookie law explained. urlhttps://www.cookielaw.org/the-cookielaw/ (2017), accessed 24-10-2017

15. Perry, T.S., Voelcker, J.: Of mice and menus: Designing the user-friendly interface. IEEE Spectr. 26(9), 46-51 (Sep 1989), https://doi.org/10.1109/6.90184

16. Refsnes, D.: Onmouseover event. urlhttps://www.w3schools.com/jsref (2017), accessed 12-09-2017

17. Reich, M.: The importance of designing for mobile first. urlhttps://www.commonplaces.com/blog/the-importance-of-designing-for-mobilefirst (2017)

18. Sears, A., Shneiderman, B.: High precision touchscreens: Design strategies and comparisons with a mouse. Int. J. Man-Mach. Stud. 34(4), 593-613 (Apr 1991), http://dx.doi.org/10.1016/0020-7373(91)90037-8

19. Shanis, J.M., Hedge, A.: Comparison of mouse, touchpad and multitouch input technologies. Proceedings of the Human Factors and Ergonomics Society Annual Meeting 47(4), 746-750 (2003), https://doi.org/10.1177/154193120304700418 